\title{
Deuterium Balmer emission from nebulae ${ }^{1}$
}

Guillaume Hébrard

Institut d'Astrophysique de Paris, CNRS, 98 bis boulevard Arago, F-75014 Paris, France

Daniel Péquignot

DAEC, Observatoire de Paris-Meudon, France

Alfred Vidal-Madjar

Institut d'Astrophysique de Paris, France

Jeremy R. Walsh

European Southern Observatory, Garching, Germany

Roger Ferlet

Institut d'Astrophysique de Paris, France

\begin{abstract}
We report on the detection and first identification of the deuterium Balmer lines $\mathrm{D} \alpha$ and $\mathrm{D} \beta$, observed in emission in the Orion Nebula (M 42). The excitation mechanism is UV fluorescence from the Lyman(D I) lines at the interface between the H II region and the molecular cloud. These lines may open the possibility to measure $\mathrm{D} / \mathrm{H}$ in galactic $\mathrm{H}$ II regions and, e.g., low-metallicity extragalactic $\mathrm{H}$ il regions, using optical spectroscopy. Fluorescence provides an extremely sensitive way to detect deuterium. Thus, the non-detection of $\mathrm{D} \alpha$ and $\mathrm{D} \beta$ in the planetary nebula NGC 6572 leads to the stringent upper limit $(\mathrm{D} / \mathrm{H})_{\mathrm{NGC}} 6572$ less than the order of $1 \times 10^{-7}$.
\end{abstract}

A detailed appraisal of the evolution of deuterium is crucial for cosmology. New methods to determine $\mathrm{D} / \mathrm{H}$ are of interest [see $e . g$. Lemoine et al. (1999) for a review]. So far, attempts to identify the Balmer lines of deuterium have been unsuccessful in any astrophysical site. We have performed a dedicated search for these lines in $\mathrm{H}$ II regions.

We have secured deep spectra of the Orion Nebula at the Canada-FranceHawaii Telescope using the Gecko spectrograph in October 1997 and September 1999. Two very narrow emission lines $\left(\mathrm{FWHM} \simeq 8 \mathrm{~km} \mathrm{~s}^{-1}\right.$ ) were detected in the blue wings of both $\mathrm{H} \alpha$ and $\mathrm{H} \beta, \sim 11 \mathrm{~km} \mathrm{~s}^{-1}$ redward of the positions expected for the deuterium Balmer lines $\mathrm{D} \alpha$ and $\mathrm{D} \beta$ in the $\mathrm{H}$ I emission line frame

\footnotetext{
${ }^{1}$ Based on observations collected at the Canada-France-Hawaii Telescope, Hawaii, USA.
} 


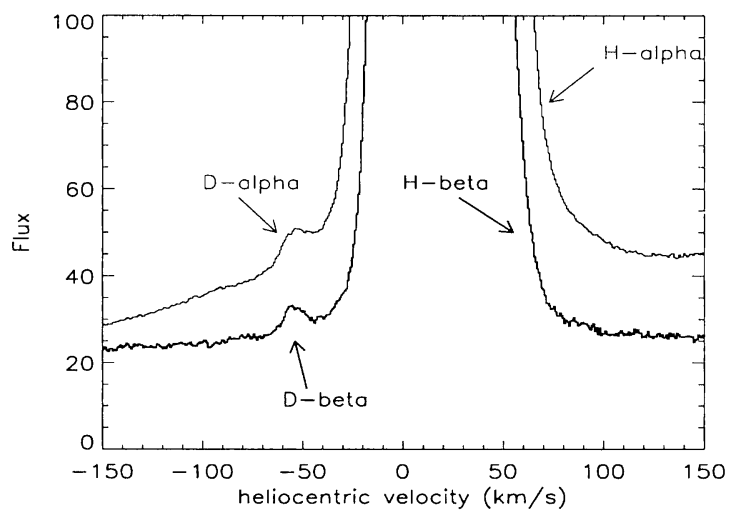

Figure 1. Detection of $\mathrm{D} \alpha$ and $\mathrm{D} \beta$ in Orion. The vertical scale corresponds to peak fluxes 7250 and 2500 for $\mathrm{H} \alpha$ and $\mathrm{H} \beta$ respectively.

(see Figure 1). It is argued (Hébrard et al. 2000) that: (1) the narrowness of the lines excludes emission from the hot $\mathrm{H}^{+}$region and therefore either $\mathrm{HI}$ or D I recombination excitation; (2) $\mathrm{H} \mathrm{I}$ emission is highly unlikely considering the very high velocity $\left(70 \mathrm{~km} \mathrm{~s}^{-1}\right)$ and velocity coherence over a large area (5'); (3) however, assuming D I emission, the radial velocity of these lines closely coincides with that of the $\mathrm{H}^{0}$ interface between the $\mathrm{H}^{+}$and $\mathrm{H}_{2}$ regions (the Photon Dominated Region); (4) then, the D I lines are likely to be excited by fluorescence following Ly(D I) radiative pumping by the stellar UV continuum, as confirmed by order of magnitude estimates. The fluxes of $\mathrm{D} \alpha$ and $\mathrm{D} \beta$ being quite similar, the next deuterium Balmer lines are likely to be detectable. A careful study of the deuterium Balmer decrement may lead to a new determination of $\mathrm{D} / \mathrm{H}$.

Using the same configuration, we obtained spectra of six planetary nebulae (PNe). We did not detect any line at the $\mathrm{D} \alpha$ and $\mathrm{D} \beta$ wavelengths. Deuterium is believed to be much depleted in PNe owing to its destruction in the parent stars. Indeed, deuterium has never been observed in PNe by whatever means. Nevertheless, our data can lead to interesting upper limits to $\mathrm{D} / \mathrm{H}$. In the case of NGC 6572 , we provisionally obtain $(\mathrm{D} / \mathrm{H})_{\mathrm{NGC}} 6572<1 \times 10^{-7}$. To our knowledge, this is the most stringent upper limit to $\mathrm{D} / \mathrm{H}$ ever found for a $\mathrm{PN}$.

We suspect that D I Balmer lines have already been detected in $\mathrm{H}$ il regions but have never been recognized as such. The present results suggest that Balmer deuteriun fluorescence is a very sensitive way to detect deuterium. This is due to the large photoexcitation cross sections involved. With the new generation of very large telescopes, it is foreseen that these lines will become detectable in low-metallicity extragalactic $\mathrm{H}$ II regions, hence possibly leading to a new determination of the primordial abundance of deuterium.

\section{References}

Hébrard, G., Péquignot, D., Vidal-Madjar, A., Walsh, J. R., \& Ferlet, R. 2000, accepted for publication in A\&A Letters (astro-ph/0002141)

Lemoine, M., Audouze, J., Ben Jaffel, L., et al. 1999, New Astronomy 4, 231 